\title{
Shallow extra mixing in solar twins inferred from Be abundances ${ }^{\star}$
}

\author{
M. Tucci Maia ${ }^{1}$, J. Meléndez $^{1}$, M. Castro ${ }^{2}$, M. Asplund ${ }^{3}$, I. Ramírez ${ }^{4}$, \\ T. R. Monroe ${ }^{1}$, J. D. do Nascimento Jr. ${ }^{2,5}$, and D. Yong ${ }^{3}$ \\ 1 Departamento de Astronomia do IAG/USP, Universidade de São Paulo, Rua do Matão 1226, Cidade Universitária, \\ 05508-900 São Paulo, SP, Brazil \\ e-mail: marcelotuccimaia@usp.br \\ 2 Departamento de Física Teórica e Experimental, Universidade Federal do Rio Grande do Norte, 59072-970 Natal, RN, Brazil \\ 3 Research School of Astronomy and Astrophysics, The Australian National University, Cotter Road, Weston, ACT 2611, Australia \\ ${ }^{4}$ McDonald Observatory and Department of Astronomy, University of Texas at Austin, Austin, TX 78712, USA \\ 5 Harvard-Smithsonian Center for Astrophysics, Cambridge, Massachusetts 02138, USA
}

Received 17 November 2014 / Accepted 10 March 2015

\section{ABSTRACT}

\begin{abstract}
Context. Lithium and beryllium are destroyed at different temperatures in stellar interiors. As such, their relative abundances offer excellent probes of the nature and extent of mixing processes within and below the convection zone.

Aims. We determine Be abundances for a sample of eight solar twins for which $\mathrm{Li}$ abundances have previously been determined. The analyzed solar twins span a very wide range of age, 0.5-8.2 Gyr, which enables us to study secular evolution of Li and Be depletion. Methods. We gathered high-quality UVES/VLT spectra and obtained Be abundances by spectral synthesis of the Be II $313 \mathrm{~nm}$ doublet.

Results. The derived beryllium abundances exhibit no significant variation with age. The more fragile Li, however, exhibits a monotonically decreasing abundance with increasing age. Therefore, relatively shallow extra mixing below the convection zone is necessary to simultaneously account for the observed $\mathrm{Li}$ and Be behavior in the Sun and solar twins.
\end{abstract}

Key words. Sun: abundances - Sun: atmosphere - Sun: evolution - Sun: interior - stars: abundances - stars: interiors

\section{Introduction}

The light elements lithium and beryllium are fragile, meaning that they are destroyed at temperatures of about $2.5 \times 10^{6} \mathrm{~K}$ and $3.5 \times 10^{6} \mathrm{~K}$, respectively (through $\alpha$ and proton captures). The observed abundances of $\mathrm{Li}$ and Be thus provide constraints for the transport of material in stellar interiors. To decrease the $\mathrm{Li}$ and $\mathrm{Be}$ abundances, the material has to be transported to deeper and hotter regions within the star before returning to the surface. In the Sun, lithium destruction requires temperatures somewhat hotter than those achieved at the base of the convective zone according to standard models of stellar evolution. The fact that the observed photospheric Li abundance is some 150 times lower than the meteoritic value thus necessitates extra mixing below the convection zone. The mechanism (or mechanisms) for depleting the light elements is still debated. Possible processes leading to extra mixing include rotation (Pinsonneault et al. 1989), internal gravity waves (Charbonnel \& Talon 2005), microscopic diffusion and gravitational settling (Michaud et al. 2004), and convective overshooting (Xiong \& Deng 2007). Because beryllium destruction requires greater temperatures than $\mathrm{Li}$, its abundance serves to constrain the extent of this extra mixing.

The solar photospheric Be abundance has been a source of contention. Early work suggested that Be was depleted in the solar photosphere compared to meteorites (Chmielewski et al. 1975). In solar-type stars, the Be abundances can only

\footnotetext{
* Based on observations obtained at the European Southern Observatory (ESO) Very Large Telescope (VLT) at Paranal Observatory, Chile (observing program 083.D-0871).
}

be estimated through the Be II doublet at $313 \mathrm{~nm}$, a spectral region difficult to analyze because of blends and uncertain atomic data. It has long been debated whether there is a substantial amount of missing UV opacity (e.g., Magain 1987; Kurucz 1992; Allende Prieto \& Lambert 2000). Balachandran \& Bell (1998) attempted to empirically calibrate the amount of missing continuous UV opacity by enforcing the same $\mathrm{O}$ abundances from the $\mathrm{OH} \mathrm{A}-\mathrm{X}$ lines near the Be doublet and the $\mathrm{OH}$ vibration-rotation lines in the infrared. They inferred a substantial amount of this missing opacity and also that the solar Be abundance is the same as the meteoritic value within errors, a conclusion which Asplund (2004) also reached using a more sophisticated 3D hydrodynamical model atmosphere. Without properly accounting for this additional continuous opacity, the Be abundance becomes underestimated, which leads to erroneous conclusions whether there even is a solar Be depletion; in fairness, we note, however, that Chmielewski et al. (1975) concluded that the uncertainty in their Be abundance $(1.15 \pm$ 0.20 dex) was too large to advocate any substantial Be depletion. These suspicions about substantial missing UV continuous opacity were subsequently confirmed by Bell et al. (2001) using new calculations by the Iron Project for the bound-free opacity of Fe I. More recently, Takeda et al. (2011) studied a sample of 118 solar analogs and suggested that Be depletion in the Sun could be significant.

There have been several observational studies of Be in solartype stars (Santos et al. 2004; Boesgaard \& Krugler Hollek 2009; Randich 2010; Takeda et al. 2011), but none focused on solar twins, except for the qualitative work by Takeda \& Tajitsu (2009) on three solar twins. The importance of solar twins is that as they 
have nearly solar mass and composition (Meléndez et al. 2014b), their evolution is similar to that of the Sun. Hence, solar twins at different evolutionary stages in the main sequence can be used as proxies of the Sun at different ages. In this work, we obtain Be abundances for solar twins in a broad age range to provide constraints on Be depletion during the main sequence and thus on the extent of any extra mixing below the convection zone.

\section{Observations and data reduction}

Spectra of eight solar twins and the Sun were obtained with the UVES spectrograph on the $8.2 \mathrm{~m}$ UT2 Very Large Telescope at ESO Paranal, on 29-30 August 2009. We used the dichroic mode, obtaining simultaneous UV and optical coverage in two setups: i) with standard settings of $346 \mathrm{~nm}+580 \mathrm{~nm}$; ii) with the standard $346 \mathrm{~nm}$ and a nonestandard setting centered at $830 \mathrm{~nm}$. We achieved a high signal-to-noise ratio $(\mathrm{S} / \mathrm{N})$ in the UV because the $346 \mathrm{~nm}$ setting (306-387 nm) was covered in both setups. The $580 \mathrm{~nm}$ standard setting covered the optical (480-682 $\mathrm{nm}$ ) region, and our $830 \mathrm{~nm}$ setting included the red region (642-1020 nm). The UV setup was used to obtain the Be abundance, the optical setup was used to obtain the stellar parameters and $\mathrm{Li}$ abundances.

We achieved a resolving power $R=65000$ at 306-387 nm and $R=110000$ for $480-1020 \mathrm{~nm}$. The solar spectrum was also obtained through the observation of the asteroid Juno with the same spectrograph setup and served as the solar reference in our differential analysis. The spectral orders were extracted and wavelength calibrated using IRAF, with additional data processing performed with IDL. The reduced spectra have $S / N \sim 100$ and 1000 near the $\mathrm{Be}$ and $\mathrm{Li}$ lines, respectively.

There are no spectral regions free from lines in the near UV spectra of solar twins. Hence, we employed a dedicated continuum normalization technique, which is described in Ramírez et al. (2008). We took advantage of the superb continuum normalization of the solar spectrum reported by Kurucz et al. (1984), which is used as a reference. Each order of the UVES spectra was divided by its corresponding piece from the spectrum of Kurucz et al. after matching their spectral resolution, correcting for radial velocity offsets, and rebinning to a common wavelength sampling. In principle, the result should have been a smooth function corresponding to the shape of the continuum (the upper "envelope") of the UVES spectra. However, because of the finite $\mathrm{S} / \mathrm{N}$ values and instrumental differences or small defects, this envelope had to be smoothed out using a 100-pixel wide $(\sim 0.16 \mathrm{~nm})$ median filter. The UVES data were then divided by this envelope in each order. In essence, this procedure makes the UVES spectra inherit the continuum normalization of the spectrum of Kurucz et al.

\section{Analysis of the Be abundances}

We used the doublet resonance lines of Be II at $313.0420 \mathrm{~nm}$ and $313.1065 \mathrm{~nm}$ to determine Be abundances; these are the only Be lines available for observation from the ground in solar-type stars. As the lines are in the UV region and blended by different species of atoms and molecules, the abundance determination was carried out by means of spectral synthesis.

Our initial line list was based on the list of Ashwell et al. (2005) and was checked with the list by Primas et al. (1997), which is the list used by Takeda et al. (2011). In some cases we modified the $\log g f$-values to achieve a good agreement with the spectrum of the Sun and of a solar analog that is
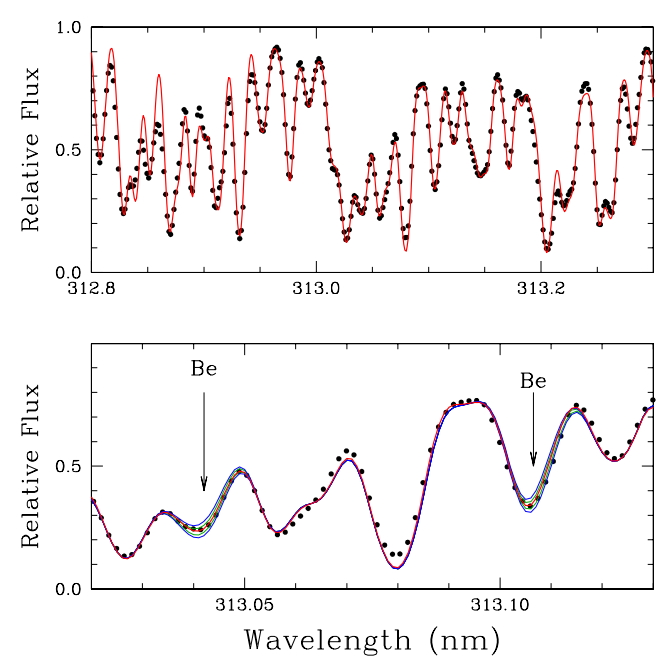

Fig. 1. Comparison between the observed (points) and synthetic (lines) spectra of the solar twin HIP 102152 around the Be II lines. The best fit is the central line, the other lines show changes in the Be abundance by \pm 0.05 and \pm 0.10 dex.

severely depleted in Be (Schirbel et al., in prep.). As the Bepoor solar analog does not show any Be lines, it was of great help to better constrain the lines blending the Be features in the Sun. We emphasize that both the Sun and the Be-depleted solar analog were observed with the same UVES setup as the solar twins and were reduced in the same way, allowing thus a reliable differential abundance analysis between the Sun and the solar twins ${ }^{1}$. In Fig. 1, we show the spectral synthesis for the solar twin HIP 102152. The weaker Be II $313.1 \mathrm{~nm}$ line is less blended and offers more reliable abundances. Since the stronger $313.0 \mathrm{~nm}$ line is heavily blended by $\mathrm{CH}$ and $\mathrm{OH}$ lines, abundances were determined for comparison, but not used in the final determination.

For the spectral synthesis we used the synth driver of the February 2014 version of the 1D local thermodynamical equilibrium (LTE) code MOOG (Sneden 1973), which includes continuum scattering. We adopted $A(\mathrm{Be})=1.38$ dex as the standard solar Be abundance (Asplund et al. 2009). The model atmospheres were interpolated from the ATLAS9 Kurucz's grid (Castelli \& Kurucz 2004) with the $T_{\text {eff }}, \log g,[\mathrm{Fe} / \mathrm{H}]$ and microturbulence determined by Monroe et al. (2013), Meléndez et al. (2014a), and Monroe et al. (in prep.). Asplund (2005) and Takeda \& Tajitsu (2009) concluded that the Be II lines are insensitive to non-LTE effects in the Sun. Considering the similarity in stellar parameters among the solar twins and the differential nature of the analysis, any differential non-LTE corrections would probably be vanishingly small (e.g., Meléndez et al. 2012), hence no corrections were applied.

To determine the macroturbulence line broadening, we first analyzed the line profiles of the Fe I $602.7050 \mathrm{~nm}, 609.3644 \mathrm{~nm}$, $615.1618 \mathrm{~nm}, 616.5360 \mathrm{~nm}, 670.5102 \mathrm{~nm}$ and Ni I $676.7772 \mathrm{~nm}$ lines in the Sun; the syntheses also included a rotational broadening of $v \sin i=1.9 \mathrm{~km} \mathrm{~s}^{-1}$ (Bruning 1984; Saar \& Osten 1997), and the instrumental broadening. The macroturbulent

1 Our modified $\log g f$-values of the Be II lines are 0.6 dex lower than those recommended by Fuhr \& Wiese (2010), but we emphasize that our values are only valid for our internal differential analysis. In other words, they are only valid when using our set of reduced UVES spectra, the 2014 version of MOOG, the set of solar abundances of Asplund et al. (2009), and the adopted blends for the Be II region. Moreover, our Be abundances are differential relative to the solar Be abundance of Asplund et al. (2009). 
Table 1. Be abundances and errors for the eight solar twins and the Sun, together with the inferred macroturbulence, $v \sin i$, and ages of the stars.

\begin{tabular}{lcccc}
\hline \hline Star & $\begin{array}{c}V_{\text {macro }} / v \sin i \\
\left(\mathrm{~km} \mathrm{~s}^{-1}\right)\end{array}$ & $\begin{array}{c}A(\mathrm{Be}) \\
(\mathrm{dex})\end{array}$ & $\begin{array}{c}\text { Param }^{a} / \mathrm{obs}^{b} / \text { total }^{c} \\
(\mathrm{dex})\end{array}$ & $\begin{array}{c}\text { Age } \\
(\mathrm{Gyr})\end{array}$ \\
\hline HD 20630 & $3.5 / 4.2$ & 1.44 & $0.03 / 0.03 / 0.04$ & $0.5_{-0.2}^{+0.2}$ \\
HD 202628 & $3.7 / 2.4$ & 1.51 & $0.01 / 0.04 / 0.04$ & $0.9_{-0.5}^{+0.5}$ \\
HIP 30502 & $3.5 / 1.6$ & 1.43 & $0.01 / 0.03 / 0.03$ & $6.0_{-0.4}^{+0.5}$ \\
HIP 73815 & $3.6 / 1.7$ & 1.49 & $0.01 / 0.03 / 0.03$ & $6.8_{-0.3}^{+0.3}$ \\
HIP 77883 & $3.4 / 1.8$ & 1.42 & $0.01 / 0.02 / 0.02$ & $7.5_{-0.3}^{+0.3}$ \\
HIP 89650 & $3.8 / 1.7$ & 1.47 & $0.01 / 0.02 / 0.02$ & $4.2_{-0.5}^{+0.3}$ \\
18Sco & $3.7 / 2.0$ & 1.52 & $0.01 / 0.03 / 0.03$ & $2.9_{-1.0}^{+1.1}$ \\
HIP 102152 & $3.5 / 1.8$ & 1.41 & $0.01 / 0.04 / 0.04$ & $8.2_{-0.3}^{+0.3}$ \\
Sun & $3.6 / 1.9$ & 1.38 & $0.00 / 0.01 / 0.01$ & 4.6 \\
\hline
\end{tabular}

Notes. ${ }^{(a)}$ Errors due to stellar parameters. ${ }^{(b)}$ Observational errors. ${ }^{(c)}$ Quadric sum of the observational and systematic errors.

velocity found for the Sun is $V_{\text {macro, } \odot}=3.6 \mathrm{~km} \mathrm{~s}^{-1}$. For the solar twins, we estimate the macroturbulence following Meléndez et al. (2012; average of Eqs. (E.2) and (E.3)): $V_{\text {macro,star }}=$ $V_{\text {macro, } \odot}+\left(T_{\text {eff }}-5777\right) / 486$.

With the macroturbulence fixed, $v \sin i$ was estimated for the solar twins sample by fitting the profiles of the six lines mentioned above, also including the instrumental broadening. Finally, the best-fitting Be abundances were estimated using a $\chi^{2}$-procedure. The estimated macroturbulence, $v \sin i$ and Be abundances for the entire sample can be found in Table 1.

We estimated the errors considering both observational and systematic uncertainties. The observational errors are due to uncertainties of the continuum placement and $\mathrm{S} / \mathrm{N}$ (the synthetic spectra were shifted vertically within the allowed noise of the observed spectrum; the abundance variation due to this shift was adopted as the observational error). For the systematic errors we considered the errors in the stellar parameters. Both observational and systematic errors were added in quadrature.

Thanks to the high internal precision of the atmospheric parameters derived in our solar twin stars, we were able to employ standard isochrone techniques to measure reliable relative ages for these objects. We adopted the ages previously derived by our group (Monroe et al. 2013, 2015; Melendez et al. 2014a) using the algorithm described in Ramírez et al. (Ramírez et al. 2013, 2014), which computes the age probability distribution function by comparing the location of the star on the $T_{\text {eff }}, \log g,[\mathrm{Fe} / \mathrm{H}]$ parameter space with the values predicted by theory. For the two youngest stars, we also used other age indicators, as described in Monroe et al. (2015). In short, we adopted a rotational age $(0.5 \pm 0.2$ Gyr $)$ for HD 20630, which excellently agrees with Ribas et al. (2010), who obtained $0.6 \pm 0.2$ Gyr using different indicators. For HD 202826 a rotation period is not available to estimate its age, hence we used its chromospheric activity, X-ray luminosity, and isochrones. From the isochrones we derive an average mass for the sample stars of $1.01 \pm 0.03 M_{\odot}$, that is, solar within the errors, reinforcing thus the use of solar twins as proxies of the Sun at different ages. The estimated ages are given in Table $1^{2}$.

\footnotetext{
2 Note that our ages are differential and that our errors are only internal. We caution that the ages may turn out to be slightly older or younger depending on the choice of isochrones, making the age range covered by the sample slightly wider or narrower, but the relative ages are reasonably well constrained, as shown for example in Fig. 5 of Meléndez et al. (2014a), where the relative ages between the Sun and the solar twin 18 Sco are consistent for two different sets of isochrones.
}

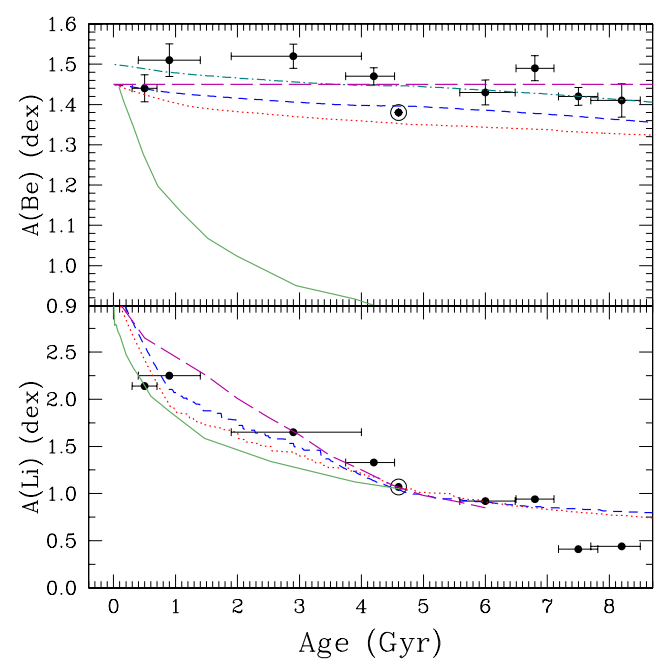

Fig. 2. Be (top) and Li (bottom) abundances vs. age. For the models of Be depletion, we adopt an initial meteoritic $A(\mathrm{Be})=1.45 \mathrm{dex}$, which is based on the value by Lodders (2003; $1.41 \mathrm{dex}$ ), plus the $0.04 \mathrm{dex}$ gravitational settling effect over $4.5 \mathrm{Gyr}$. The models of Li depletion were normalized to the solar Li abundance. The green solid lines are the models by Pinsonneault et al. (1989), the red dotted lines represent the models by do Nascimento et al. (2009), the blue dashed lines are the modified models of do Nascimento et al. (2009; see text), and the purple long-dashed lines are predictions of Be depletion by Xiong \& Deng (2007) and Li depletion by Xiong \& Deng (2009). The teal dotted-dashed line (top panel) is the same Be depletion model of do Nascimento et al. (2009) shown by the red dotted lines, but with a higher initial $A(\mathrm{Be})=1.50 \mathrm{dex}$ (rather than $A(\mathrm{Be})=1.45 \mathrm{dex})$. The additional 0.05 dex is to compensate for the refractory depletion of $\mathrm{Be}$ in the Sun (see text).

\section{Discussion}

Figure 2 (top panel) shows the measured Be abundances in our solar twins as a function of stellar age. The scatter in the Be content is very small: 0.04 dex. This is significantly less than in previous studies; for example, Takeda et al. (2011) analyzed $\mathrm{Be}$ in a large sample of solar analogs and found a dispersion of 0.2 dex. A linear fit to our data using the error bars in both age and Be abundances gives a slope of $-8.09 \times 10^{-3} \pm$ $4.17 \times 10^{-3} \mathrm{dex} / \mathrm{Gyr}$, which is a shallow trend at best. For comparison, we show in the bottom panel the corresponding non-LTE $\mathrm{Li}$ abundances for the same sample stars (Monroe et al. 2015). A fit to the Li data gives a slope of $-0.23 \pm 0.01 \mathrm{dex} / \mathrm{Gyr}$. Clearly, there is a steep Li depletion, but the mixing processes responsible for the destruction of lithium must be relatively shallow and cannot transport material to deeper regions where significant Be destruction can occur.

In standard evolution models of the Sun, the depletion of the light elements $\mathrm{Be}$ and $\mathrm{Li}$ is expected to occur only below the convective zone and thus the surface abundances should remain unchanged, obviously in contrast to the observational evidence in the case of Li. Only for less massive stars do standard stellar models predict significant Be depletion as a result of the deeper convective zone (Santos et al. 2004). Owing to the monotonically increasing Li depletion with age (Monroe et al. 2013), most Li destruction occurs on secular timescales during the main sequence. Since standard models cannot predict this behavior, there must be extra mixing below the convection zone that brings material down to sufficiently large depths and temperatures, and then back into the convection zone. In Fig. 2, four different predictions for $\mathrm{Be}$ and $\mathrm{Li}$ depletion are compared. The model by Pinsonneault et al. (1989) considers rotationally induced mixing 
and depletes Li reasonably well, but depletes far too much Be (green solid line). The model of do Nascimento et al. (2009) includes extra mixing due to diffusion (including gravitational settling) and rotation. It reproduces $\mathrm{Li}$ in solar twins well, but Be depletion is somewhat larger than observed (red dotted lines). We have modified this model to calibrate the amount of meridional circulation, achieving a steep and a shallow $\mathrm{Li}$ and $\mathrm{Be}$ depletion, respectively (blue dashed lines). The new model has meridional circulation with a lower efficiency, hence destroying less $\mathrm{Be}$ and $\mathrm{Li}$, and we increased the turbulent diffusion coefficient of the tachocline below the convective zone to destroy more Li (without affecting Be). Finally, the models of Xiong \& Deng $(2007,2009)$ incorporate convective overshoot as well as gravitational settling. They reproduce the Li depletion with age but do not deplete Be (purple long-dashed line), unlike the observations, which seems to suggest a shallow depletion of Be.

The initial (zero-age) Be abundance for the model predictions assumes them to be equal to the meteoritic values. For the meteoritic abundance, Lodders (2003) recommended $A(\mathrm{Be})=$ $1.41 \pm 0.08 \mathrm{dex}$, which was subsequently revised to $A(\mathrm{Be})=$ $1.32 \pm 0.03$ dex by Lodders et al. (2009). These are indirect measurements, however, because Be is difficult to measure in carbonaceous chondrites of type CI, which are the least modified meteorites and thus the preferred choice when inferring the primordial solar system abundances. Instead, the meteoritic value was estimated from the relative abundances of refractory elements in CM and CV chondrites in which Be has been measured in a couple of cases. The relatively large uncertainty for Be for being meteorites reflects this indirect procedure.

Since the absolute abundance scales for meteorites are set by enforcing that the photospheric and meteoritic Si abundances are equal (Asplund 2000; Asplund et al. 2009) and all elements heavier than hydrogen have experienced gravitational settling in the Sun over the past 4.5 Gyr (see discussion in Asplund et al. 2009), a more appropriate initial Be value for these model predictions would thus be 0.04 dex higher than the abundances recommended by Lodders (2003) and Lodders et al. (2009). Our solar twins data would seem to suggest that the higher meteoritic Be abundance (1.41 dex) is more appropriate. We thus added 0.04 dex to this value, adopting $A(\mathrm{Be})=1.45 \mathrm{dex}$ as the initial Be abundance in Fig. 2. The Sun may be slightly less abundant in Be by $\sim 0.05$ dex for its age compared with other similar solar twins (Fig. 2), but a larger number of solar twins would be required to confirm this impression. Perhaps the somewhat lower solar Be abundance could arise simply because the Sun is poor in refractories (Meléndez et al. 2009); Be has a condensation temperature of $T_{\text {cond }}=1452 \mathrm{~K}$ (Lodders 2003), which means that it is a refractory element. The Sun is probably deficient in refractories as a result of the formation of rocky planets in the solar system (Meléndez et al. 2009). For its $T_{\text {cond }}$, we estimate that Be should be depleted in $\sim 0.05$ dex in the Sun. Interestingly, if we consider an initial $A(\mathrm{Be})=1.5 \mathrm{dex}$ owing to the refractory depletion of $\mathrm{Be}$, the modified model by do Nascimento et al. (2009) reproduces the Be abundances of most solar twins well (dotted-dashed line in Fig. 2).

Recently, Adibekyan et al. (2014) suggested that the depletion of refractory elements in the Sun relative to solar twins could be an age effect. If this interpretation is correct, and because $\mathrm{Be}$ is a refractory element, we should have found that beryllium in solar twins older than the Sun $(4.6 \mathrm{Gyr})$ is depleted relative to the Sun, because Adibekyan et al. (2014) analyzed their solar analogs relative to the Sun. However, this is not what we observe in Fig. 2. Thus, our results seem to be in conflict with the interpretation by Adibekyan et al. (2014).

\section{Conclusions}

We presented the first detailed study of beryllium abundances in solar twins covering a broad range of ages (0.5-8.2 Gyr). Our analysis revealed that the Be abundance is relatively constant with age, with a scatter of only 0.04 dex and a weak, if any, trend with age. This is in contrast to the large observed depletion of Li with age (Baumann et al. 2010; Monroe et al. 2013; Meléndez et al. 2014b), showing that the transport mechanisms are deep enough to reach the region where $\mathrm{Li}$ is burned, but not deep enough to reach the higher temperatures needed to burn Be. Our Li and Be results provide stringent constraints on stellar models and nonstandard mixing processes beyond treating convection through the mixing length theory.

Acknowledgements. We thank Johanna F. Jarvis for sharing her Be line list. M.T.M. thanks for support by CNPq (142437/2014-0). J.M. thanks for support by FAPESP (2012/24392-2). M.A. and D.Y. acknowledge financial support from the Australian Research Council (grant DP120100991).

\section{References}

Adibekyan, V. Z., González Hernández, J. I., Delgado Mena, E., et al. 2014, A\&A, 564, L15

Allende Prieto, C., \& Lambert, D. L. 2000, AJ, 119, 2445

Asplund, M. 2000, A\&A, 359, 755

Asplund, M. 2004, A\&A, 417, 769

Asplund, M. 2005, ARA\&A, 43, 481

Asplund, M., Grevesse, N., Sauval, A. J., \& Scott, P. 2009, ARA\&A, 47, 481

Ashwell, J. F., Jeffries, R. D., Smalley, B., et al. 2005, MNRAS, 363, L81

Balachandran, S. C., \& Bell, R. A. 1998, Nature, 392, 791

Baumann, P., Ramírez, I., Meléndez, J., Asplund, M., \& Lind, K. 2010, A\&A, 519, A87

Bell, R. A., Balachandran, S. C., \& Bautista, M. 2001, ApJ, 546, L65

Boesgaard, A. M., \& Krugler Hollek, J. 2009, ApJ, 691, 1412

Bruning, D. H. 1984, ApJ, 281, 830

Castelli, F., \& Kurucz, R. L. 2004 [arXiv: astro-ph/0405087]

Charbonnel, C., \& Talon, S. 2005, Science, 309, 2189

Chmielewski, Y., Brault, J. W., \& Mueller, E. A. 1975, A\&A, 42, 37

do Nascimento, J. D., Jr., Castro, M., Meléndez, J., et al. 2009, A\&A, 501, 687

Fuhr, J. R., \& Wiese, W. L. 2010, J. Phys. Chem. Ref. Data, 39, 013101

Kurucz, R. L. 1992, Rev. Mex. Astron. Astrofis., 23, 181

Kurucz, R. L., Furenlid, I., Brault, J., \& Testerman, L. 1984, National Solar Observatory Atlas, Sunspot (New Mexico: National Solar Observatory) Lodders, K. 2003, ApJ, 591, 1220

Lodders, K., Palme, H., \& Gail, H.-P. 2009, Landolt Börnstein, 44

Magain, P. 1987, A\&A, 181, 32

Meléndez, J., Asplund, M., Gustafsson, B., \& Yong, D. 2009, ApJ, 704, L66

Meléndez, J., Bergemann, M., Cohen, J. G., et al. 2012, A\&A, 543, A29

Meléndez, J., Ramírez, I., Karakas, A. I., et al. 2014a, ApJ, 791, 14

Meléndez, J., Schirbel, L., Monroe, T. R., et al. 2014b, A\&A, 567, L3

Michaud, G., Richard, O., Richer, J., \& VandenBerg, D. A. 2004, ApJ, 606, 452

Monroe, T. R., Meléndez, J., Ramírez, I., et al. 2013, ApJ, 774, L32

Pinsonneault, M. H., Kawaler, S. D., Sofia, S., \& Demarque, P. 1989, ApJ, 338, 424

Primas, F., Duncan, D. K., Pinsonneault, M. H., Deliyannis, C. P., \& Thorburn, J. A. 1997, ApJ, 480, 784

Ramírez, I., Allende Prieto, C., \& Lambert, D. L. 2008, A\&A, 492, 841

Ramírez, I., Allende Prieto, C., \& Lambert, D. L. 2013, ApJ, 764, 78

Ramírez, I., Meléndez, J., Bean, J., et al. 2014, A\&A, 572, A48

Randich, S. 2010, IAU Symp., 268, 275

Ribas, I., Porto de Mello, G. F., Ferreira, L. D., et al. 2010, ApJ, 714, 384

Saar, S. H., \& Osten, R. A. 1997, MNRAS, 284, 803

Santos, N. C., Israelian, G., Randich, S., García López, R. J., \& Rebolo, R. 2004, A\&A, 425, 1013

Sneden, C. A. 1973, Ph.D. Thesis (University of Texas)

Takeda, Y., \& Tajitsu, A. 2009, PASJ, 61, 471

Takeda, Y., Tajitsu, A., Honda, S., et al. 2011, PASJ, 63, 697

Xiong, D.-R., \& Deng, L.-C. 2007, Chin. Astron. Astrophys., 31, 244

Xiong, D. R., \& Deng, L. 2009, MNRAS, 395, 2013 